\title{
Unidad Clínico Forense: Experiencias y percepciones del equipo de salud respecto a su implementación en el hospital Gustavo Fricke
}

\author{
Clinical Forensic Unit: Experiences and perceptions of the healthcare \\ team regarding its implementation at the Gustavo Fricke Hospital
}

\author{
Rodrigo Neira ${ }^{1}$, Lucía Flores ${ }^{2}$, Francisca Valdés ${ }^{3}$
}

\begin{abstract}
${ }^{1}$ Matrón; Prof.Asistente Departamento de Promoción de la Salud de la Mujer y el Recién Nacido, Universidad de Chile; Profesional Programa Nacional de Salud de la Mujer, Subsecretaría de Salud Pública, Ministerio de Salud de Chile. ${ }^{2}$ Licenciada en Obstetricia y Puericultura, Escuela de Obstetricia, Facultad de Medicina Universidad de Chile, Chile. ${ }^{3}$ Matrona, Servicio de Neonatología, Hospital San Juan Dios, Santiago, Chile.
\end{abstract}

*Autor para correspondencia: rneirac@uchile.cl

RECIBIDO: 27 de Junio de 2020

APROBADO: 06 de Septiembre de 2020

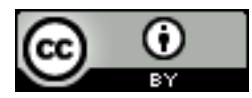

DOI: 10.22370/rev.mat.1.2020.2319

LOS AUTORES DECLARAN NO TENER CONFLICTO DE INTERESES

Palabras claves: Norma técnica, violencia sexual, medicina forense, medicina legal, política pública. Key words: Clinical guideline, sexual violence, forensic medicine, legal medicine, public policy.

\section{RESUMEN}

En Chile, en el año 2016 fue publicada la "Norma general técnica para la atención de víctimas de violencia sexual", la cual busca dar respuesta al fenómeno de la violencia sexual, estandarizando la atención, peritaje y manejo de las evidencias, a través de la formación de Unidades Clínico Forense en los servicios de salud, tomando un rol colaborador en la recopilación y manejo de evidencias, además de la atención de salud de la víctima. Si bien se ha dispuesto de recursos para el mejoramiento e implementación de nuevas Unidades Clínico Forense en la red de salud, no existen datos accesibles sobre su instauración y desarrollo. Objetivo: Describir desde la perspectiva del equipo de salud, las experiencias en la implementación de la norma para la atención de víctimas de violencia sexual en la Unidad Clínico Forense del Hospital Gustavo Fricke, en el año 2019. Material y método: Estudio cualitativo, exploratorio, aplicando una entrevista semiestructurada, a los integrantes de la Unidad Clínico Forense, con un análisis narra- tivo de contenido, construyendo categorías acordes con los hallazgos. Resultados: La implementación de la Unidad Clínico Forense se vio facilitada por la presencia de un fuerte liderazgo, la motivación personal del equipo, y la autogestión. Sin embargo, los recursos y gestiones proporcionados por los agentes gubernamentales de salud fueron percibidas como insuficientes, comprometiendo la calidad de la atención entregada, y el bienestar emocional del equipo. Conclusiones: Pese a las dificultades percibidas durante la implementación de la Unidad Clínico Forense, es considerado por sus miembros como un proceso exitoso.

\section{ABSTRACT}

In Chile, in 2016, the "General clinical guideline for the care of victims of sexual violence" was published, which seeks to respond to the phenomenon of sexual violence, standardizing care, expert reports, and the handling of evidence, through the training of Clinical Forensic Units in health services, who 
take a collaborative role in the collection and handling of evidence, in addition to the health care of the victim. Although resources have been provided for the improvement and implementation of new Clinical Forensic Units along the health network, there is no accessible data on their development and implementation. Objective: To describe from the perspective of the healthcare team, the experiences in the implementation of the general technical standard for the care of victims of sexual violence in the Forensic Clinical Unit of the Gustavo Fricke Hospital. Materials: A qualitative, exploratory study, applying a semi-structured interview to the members of the Clinical Forensic Unit, and a narrative content analysis, constructing categories according to the findings. Results: The implementation of the Clinical Forensic Unit was facilitated by the presence of strong leadership, the personal motivation of its members, and self-management. However, resources and management provided by the government health agents were perceived as insufficient, compromising the quality of the attention delivered and the emotional well-being of the team. Conclusions: Despite the difficulties presented during the implementation of the Clinical Forensic Unit, it is perceived by its members as a successful process.

\section{INTRODUCCIÓN}

La violencia sexual es un fenómeno sensible y complejo en todo el mundo, por los desafíos que conlleva su manejo teórico y práctico tanto por el estado como para la sociedad en su conjunto. La Organización Mundial de la Salud (OMS) define a la violencia sexual como: "Todo acto sexual, la tentativa de consumar un acto sexual, los comentarios o insinuaciones sexuales no deseados, o las acciones para comercializar o utilizar de cualquier otro modo la sexualidad de una persona mediante coacción por otra persona, independientemente de la relación de ésta con la víctima" (1).

La violencia sexual se enmarca dentro de un orden social y cultural, donde los mitos y estereotipos alrededor de lo femenino y lo masculino suponen herramientas de silenciamiento e invisibilización de la violencia (2), situando a las mujeres en diversos contextos desventajosos. Por otro lado, aunque les afecta con menor frecuencia, la violencia sexual hacia los hombres asociado a factores vinculados a la masculinidad tradicional obstaculizan el reconocimiento de la experiencia abusiva en los varones (3), quienes también sufren consecuencias físicas y psicosociales.

Un segundo grupo de la sociedad que se encuentra vulnerable debido a sus características intrínsecas son los niños y las niñas. La violencia sexual infantil se define como "el contacto o interacción de un niño, niña o adolescente en actividades sexuales no acorde con su nivel evolutivo, es decir, al desarrollo emocional, cognitivo o social esperado para su edad, pudiendo ocurrir con o sin contacto físico" (4).

De acuerdo a la Encuesta Nacional de Victimización por Violencia Intrafamiliar y Delitos Sexuales del 2013, el 31,9\% de las de las mujeres en Chile ha sufrido alguna forma de violencia por sus familiares, pareja o ex pareja, donde el 6,3\% ha sido de carácter sexual (5).

En el caso de niños y niñas, el 7,3\% declaran haber sido tocados o acariciados sexualmente contra su voluntad o haber sido obligados a tocar sexualmente o a realizar alguna actividad de contenido sexual. El promedio de edad a la cual ocurre el primer abuso sexual es a los 10,5 años. El 87,3\% de los agresores de delitos sexuales a los niños y niñas fue un hombre, y en cuanto a la relación de la víctima con su agresor, el $39,9 \%$ fue algún familiar y el $45,6 \%$ un conocido (5).

De este modo, que el fenómeno de la violencia sexual se encuentre instalado desde las estructuras socioculturales demanda un gran esfuerzo hacia el Estado, y a la sociedad (6). Así, los mejores métodos para combatir la violencia sexual requieren la cooperación de diversos sectores, como los de la salud, de la educación, de bienestar social y de justicia penal, poniendo énfasis en la prevención, velando al mismo tiempo porque las víctimas de violencia tengan acceso a servicios y apoyo apropiados (1).

En Chile se han llevado a cabo múltiples iniciativas en relación a la atención de víctimas de violencia sexual. Estas comienzan en el año 1953 con los peritajes sexológicos en el Servicio Médico Legal (SML). Más tarde, en el año 1995, se implementa el sistema de turnos de 24 horas en sexología forense para la Región Metropolitana (7). Posteriormente, nacen nuevos enfoques que buscan satisfacer la 
necesidad de atención en salud de la víctima, como la iniciativa de las Salas de Acogida (Salas Thuthuzela) en los hospitales públicos del Chile en el año 2005, sin resultados exitosos, en parte por la falta de formación y sobrecarga asistencial del recurso humano destinado para este fin (8). En el año 2014, tras la creación del "Consejo Nacional de la Infancia y Adolescencia", se genera una mesa de trabajo entre el Ministerio Público, Servicio Médico Legal, la UNICEF y el Ministerio de Salud, cuyo interés común fue diseñar un componente transversal e intersectorial para brindar una atención integral y oportuna a las personas que han sido víctimas de violencia sexual (8). Fruto del mencionado trabajo intersectorial, el año 2016 fue publicada la "Norma general técnica para la atención de víctimas de violencia sexual" la cual fue creada en base a la revisión y actualización de la normativa vigente.

Esta norma define como su objetivo general "Estandarizar la atención, peritaje y manejo de las evidencias en las personas que han sufrido violencia sexual, a través de la definición de un procedimiento único para todos los profesionales y técnicos que intervienen en el proceso", consolidándose a través de las Unidades Clínico Forense (UCF), las cuales son direccionadas a través de la Subsecretaría de Redes Asistenciales del Ministerio de Salud (8). Estas unidades se crean con la intención de que representen la puerta de entrada para las víctimas de violencia sexual, tanto a la atención en salud, como al proceso penal, brindando una atención integral, disminuyendo la victimización secundaria y realizando pericias de calidad, tomando un rol colaborador en la recopilación y manejo de evidencias.

Por política pública entendemos la acción gubernamental que moviliza recursos humanos, financieros e institucionales para resolver problemas públicos dentro de una sociedad. La formulación de la política pública comienza desde que un asunto se concibe como problema en la agenda pública (9). Por otro lado, la implementación es el conjunto de acciones que se establecen para lograr los objetivos propuestos; debe ser planificada desde la formulación de la política pública y es considerada la fase crítica en el éxito, ya que es complejo establecer una coordinación colectiva armónica y efectiva (10).
Existen elementos que pueden incidir en el $\mathrm{co}^{-}$ rrecto desarrollo de este documento ministerial como la falta de recursos humanos y/o económicos, infraestructura inadecuada, escasa capacitación de los profesionales, o incluso deficiencias en la misma normativa regulatoria, sin embargo, el factor más preponderante es la disposición de los actores involucrados, esto justificado en la presencia de intereses contradictorios, percepciones distintas de los actores en distintos lugares de la implementación y resistencia al cambio (10).

Si bien entre los años 2014 y 2017 se ha dispuesto de recursos al Servicio Médico Legal y al Ministerio de Salud para el mejoramiento de las capacidades ya instaladas y la implementación de 16 UCF en la red de salud (11), no existen datos accesibles sobre el desarrollo y monitorización de estas en los servicios de urgencia, generando desconocimiento en la población sobre dónde acudir ante este tipo de delitos.

En vista de lo anterior, existe la necesidad monitorizar la existencia de las UCF en la red de salud para garantizar el acceso a una atención de altos estándares a las víctimas de violencia sexual, siendo relevante indagar en aquellas UCF ya establecidas, sobre sus experiencias y percepciones durante su implementación, y así entregar directrices a otros centros de salud donde aún no se han desarrollado.

El objetivo general de la presente investigación es describir desde la perspectiva del equipo de salud, las experiencias en la implementación de la norma para la atención de víctimas de violencia sexual en la Unidad Clínico Forense del Hospital Gustavo Fricke, en el año 2019. Los objetivos específicos son: explorar las experiencias del equipo de salud en la implementación de la Unidad Clínico Forense desde su propia perspectiva y describir las barreras y facilitadores en la implementación de la Unidad Clínico Forense desde las vivencias de los trabajadores involucrados.

\section{MATERIAL Y MÉTODOS}

Se utilizó un diseño cualitativo, exploratorio con enfoque fenomenológico, como primer acercamiento al tema, con el fin de plantear nuevas líneas de investigación. La información fue recolectada en el hospital Dr. Gustavo Fricke, ubicado en la Región de Valparaíso, siendo el establecimiento de mayor com- 
plejidad de la Red del Servicio de Salud Viña del Mar Quillota (12), y el cual tiene implementada la Norma para la atención de víctimas de violencia sexual desde el año 2017, convirtiéndose en el primer establecimiento en contar con una UCF hospitalaria (13), funcionando de lunes a viernes desde 8:00 hasta 17:00 horas y localizado independiente de los servicios de urgencia.

Se presentó y obtuvo la aprobación del Comité de Ética de investigación en Seres Humanos de la Universidad de Chile, y de la Unidad Asistencial Docente del Hospital Gustavo Fricke.

No se utilizó una muestra, ya que se entrevistó a la totalidad del universo, correspondiente a 5 miembros pertenecientes a la UCF del Hospital Gustavo Fricke, siendo estos 2 médicos pediatras, 1 matrona, 1 técnico en enfermería y 1 administrativa. La unidad de análisis fueron los relatos generados por los trabajadores de la UCF que cumplieron con los siguientes criterios:

- Criterios de inclusión: Trabajadores que participaron en la implementación de la UCF.

- Criterios de exclusión: Trabajadores que llevasen un tiempo menor a 6 meses en la UCF.

Para la recolección de datos se realizó una entrevista semiestructurada a cada uno de los miembros de la UCF que cumplieran con los criterios anteriormente mencionados. Previamente, se hizo entrega a cada participante de un consentimiento informado que incluyó el objetivo de esta investigación, la metodología, la condición de participación voluntaria y confidencial así como la posibilidad de retirarse en cualquier momento.

Respecto a los criterios de rigor, la confirmabilidad, se salvaguardó por medio de la transcripción textual de las entrevistas, lo cual se complementó con el criterio de dependencia por medio de la revisión de esta por dos investigadores, asignando una codificación a cada participante. Para aumentar la credibilidad se utilizó el método de auditoría externa (14), mostrando el método y proceso de recolección de datos al investigador principal. Se utilizó un análisis narrativo de contenido en forma manual, donde se construyeron categorías acordes con los hallazgos. Al finalizar el estudio, las grabaciones fueron eliminadas de todos los dispositivos utilizados.

\section{RESULTADOS}

Se agruparon los resultados según categorías y subcategorías: 1) Inicios de la implementación, 2) Primeras experiencias y percepciones y 3) Evaluación del equipo sobre la implementación. Se observó la progresiva repetición de las respuestas alcanzando la saturación de datos.

Los entrevistados se distinguen como cinco sujetos: Entrevistado 1, Entrevistado 2, Entrevistado 3, Entrevistado 4 y Entrevistado 5. Debido al reducido tamaño de la muestra, limitamos la caracterización de los participantes para proteger la confidencialidad. A su vez, los nombres propios mencionados se reemplazaron por el cargo correspondiente.

Todos los entrevistados tienen un promedio de tiempo trabajando en la UCF de dos años y seis meses, es decir, desde los inicios de la unidad. Cada uno ha sido capacitado acerca de temáticas relacionadas con violencia sexual. Dos de los entrevistados refieren no haber tenido ninguna experiencia laboral con víctimas de violencia sexual previo a su trabajo en la UCF.

\section{1) Inicios de la implementación}

Los participantes de forma unánime relatan que la UCF nace el año 2017 tras la publicación de la Norma Técnica y la delegación de su implementación desde la directiva del hospital a la fundadora y jefa del equipo. Es ella quien inicia las gestiones para su instauración, considerando aspectos administrativos, de infraestructura, gestión de insumos y personal, adecuando la Unidad según lo detallado en la Norma Técnica. Se destaca la motivación personal de la fundadora como motor fundamental para la implementación.

a. Niveles de gestión involucrados: La creación de la UCF se adjudica principalmente a la Jefa de la unidad, además se describen agentes como el Ministerio de Salud como coordinadores de las UCF a lo largo del país; el Servicio de Salud Viña del mar-Quillota a través de una gestora, encargada de la comunicación con la unidad y entrega de recursos e insumos; la directiva del hospital y las gestiones de los mismos miembros de la 
UCF de forma particular, principalmente en la obtención y administración de insumos.

b. Factores dificultantes: Se relata la falta de recursos, insumos y personal médico en los inicios de la unidad, al ser compleja la comunicación con la gestora del Servicio de Salud Viña del Mar-Quillota, ya que desconocía los insumos médicos requeridos por la unidad. Esto debió ser compensado, a través de las gestiones de la propia unidad para la adquisición de los insumos con otras unidades u hospitales. Asimismo, fue nombrado por 2 entrevistados las escasas capacitaciones brindadas por el hospital, tanto para la matrona de la unidad, como para la TENS y administrativa, debiendo autogestionar la búsqueda de formación para el correcto desarrollo de sus funciones en la UCF.

"Algunas cosas incómodas como tener que conseguirse insumos, buscar capacitaciones porque a veces el hospital no nos capacitaba (...) Le pedía apoyo a la gestora y eso fue complejo porque ella ...sin desmerecer obviamente, es trabajadora social entonces ella tenía que comprar ciertos insumos y no me entendía, finalmente como que ella se hizo aparte un poco y tuve que yo coordinar todo" (Entrevistado 2)

Un segundo elemento descrito es que, si bien la norma es considerada por los participantes como una valiosa guía general, dos participantes refieren que existe falta de información en ciertas áreas, uno de los entrevistados menciona que a nivel pericial existían deficiencias.

"Muchas cosas salían en la norma técnica pero no eran tan específicas, o hay cosas que salen en la norma técnica pero que en la práctica no se realizan entonces... hubo muchas cosas que ir aprendiendo (...) fuimos consolidando algunos conceptos de como tomar ciertos exámenes, o más específicos." (Entrevistado 2)

Un tercer factor relatado fue el desconocimiento de los mismos funcionarios del hospital sobre la existencia y funciones de la UCF, además de la percepción de un trato hostil hacia el equipo desde el entorno hospitalario, por desempeñar sus funciones exclusivamen- te en la unidad, existiendo incluso cierto nivel de resistencia a la contratación de personal exclusivo para esta tarea.

“...sentimos que el entorno no es amigable con nosotros, el entorno hospitalario, salvo algunas personas. Como que se nos vuelve agresivo, de repente me incomoda porque tengo que proteger al equipo de que nos obliguen a hacer cosas, o se quejen porque no hacemos cosas" (Entrevistado 4).

c. Factores facilitadores: Se relata principalmente la motivación personal de la fundadora de la unidad y sus esfuerzos por el desarrollo de esta, no solo en sus gestiones administrativas, sino también por su fuerte sentido de liderazgo como jefa de la unidad, cohesionando exitosamente a los miembros de esta, fomentando el autocuidado y favoreciendo las posibilidades de formación de sus integrantes.

Otro elemento descrito por los participantes es que la presencia de funcionarios propios y exclusivos para la unidad clínico forense amplía la disponibilidad de la unidad ante la llegada espontánea de usuarios que requieran la atención.

"Debía ser un equipo exclusivo para la unidad clínico forense y no que viniera desde la urgencia "de llamado", porque por un lado la urgencia ya se ve sobrepasada con la cantidad de pacientes que hay, y por otro lado no se sabe si esos profesionales se encuentran preparados para atender a víctimas de violencia sexual" (Entrevistado 5).

A su vez, los participantes valoran las gestiones realizadas tanto por la dirección del hospital como por el Ministerio de Salud, a través de jefaturas y coordinadores ministeriales quienes, a través de videoconferencias con las distintas unidades a lo largo de Chile, resolvieron dudas sobre la Norma, unificaron conceptos y recibieron retroalimentación sobre ajustes que deberían hacerse a la norma en un futuro. 


\section{2) Primeras experiencias y percepciones}

La percepción de los entrevistados sobre el proyecto en sus orígenes es unánimemente positiva, valorando la importancia de la privacidad en la atención de las víctimas al tener un lugar exclusivo. Por otro lado, un entrevistado describe la dificultad que significaba la poca experiencia del equipo al ser la primera unidad en Chile.

El equipo describe experimentar un fuerte sentimiento de motivación por su trabajo, especialmente al ver los resultados, evidenciado en la evolución positiva de las víctimas luego de la atención, o en las sentencias entregadas a los victimarios, las cuales representarían el resultado de sus esfuerzos por entregar una atención y peritajes de alto nivel.

"Fue un desafío personal, lo vi como un desafío que cubriría una necesidad ignorada y también tal vez ocultada" (Entrevistado 5).

"He recibido sentencias de juicios, ahi te puedo decir que personalmente tengo una satisfacción, vale la pena el esfuerzo que se hace o el trabajo, en la formación, hacer los trámites, preocuparte que los informes vayan bien, que lleguen a las fechas, que las muestras no se extravien, no sé po, no les pase nada, no se dañen en el proceso." (Entrevistado 1).

a. Primeras atenciones: Se describen los primeros encuentros con los usuarios como episodios "fuertes" y "difíciles". Sin embargo, prevalece el sentimiento de percibir ser "un aporte para la atención de las víctimas", y "estar haciendo lo correcto", como una forma de asimilar los primeros sentimientos mencionados. Se relata que al obtener experiencia, adquirieron más capacidades para enfrentar estos episodios. Sin perjuicio de lo anterior, relatan nunca sentir acostumbramiento a estas experiencias.

b. Carga psicológica en el equipo: Los participantes relatan que su trabajo implica una fuerte carga psicológica por lo que significa intrínsecamente el proceso de atención de víctimas de violencia sexual. Este aspecto ha sido autogestionado como equipo, a través de jornadas de autocuidado, utilizando métodos como el defusing posterior a cada atención y de jornadas de autocuidado. No obstante, son consideradas como escasas las medidas de protección que nacen desde el hospital, pese a ser solicitado y que aparezca explícitamente en la norma.

"-Los jefes de la unidad- Siempre se han preocupado harto de nosotros, de saber que nuestra pega es dura, es terrible en realidad estar escuchando relatos... siempre han estado muy presentes en que nosotros estemos bien, en que no nos afecte, que no lleguemos a un estrés laboral solamente de cansancio mental, entonces la gestión de ellos es súper importante porque ellos están con nosotros, están en el día a día, saben lo que hacemos (...) Nosotros lo hacemos como grupo acá... porque en realidad en el hospital no hay mucho cuidado de sus trabajadores." (Entrevistado 3)

\section{3) Evaluación del equipo sobre la implementa- ción:}

La evaluación general es positiva, ya que consideran que la norma es una buena guía general para estructurar la atención y que el estándar ha mejorado progresivamente con la experiencia. Sin embargo, declaran sentir insatisfacción con las gestiones iniciales para el funcionamiento de la unidad, asociado a las instituciones de la red de gestión.

a. Elementos que han mejorado: Los participantes consideran de forma unánime a la experiencia como un factor fundamental para el perfeccionamiento de la UCF, mejorando el orden de la atención, la rapidez, el manejo de documentos y los resguardos para el confort del usuario, relacionándolo también, con la capacitación de sus miembros. Cabe señalar que uno de los entrevistados menciona la mejora en las relaciones humanas del equipo.

b. Elementos por mejorar: Se menciona la importancia de aumentar las horas de atención y la cantidad de personal para transformar a la UCF en un servicio " $24 / 7$ ", de esta forma asegurar que todos los usuarios reciban una atención y peritaje de alto estándar, lo cual se ve limitado por los escasos recursos humanos y materiales. 
Además, describen la necesidad de mejorar la comunicación con el Servicio de Salud de la región y así gestionar los recursos según las necesidades explicitadas por la Unidad.

Se describió también, la sensación de desconocimiento de la población sobre la existencia de la UCF, y por lo tanto, la necesidad de generar mayor difusión en la región. No obstante, reconocen un aumento considerable de pacientes año a año.

“ (...) Y no hay conocimiento...de la gente, de los mismos funcionarios, del público... no existe, a pesar de que llevamos 2 años, todavía, "¿Qué es una unidad clínico forense?" en vez de ser como un servicio de urgencia que todos lo conocen... nada, acá como que no existimos" (Entrevistado 1)

Existe consenso entre los entrevistados que la UCF estudiada cumple los objetivos establecidos en la norma. Un entrevistado hace hincapié en las deficiencias de la red para la contención psicológica del usuario. Por otro lado, los entrevistados consideran importante el perfeccionamiento de esta norma, valorando para su revisión las experiencias de los miembros de la unidad durante el trabajo cotidiano.

"Tiene que haber una red donde la víctima llega a la unidad, se hace el peritaje pero luego tiene que tener su apoyo psicológico, tiene que tener su reparación (...) pero eso no ocurre hoy, tenemos demora de 3 semanas, entonces necesitamos que una víctima sea contenida ahora ya, pero considero que sí, cumple con los objetivos de evitar la victimización secundaria por sobre todas las cosas, eso es lo más importante" (Entrevistado 2)

"Si bien la norma está siendo revisada y actualizada, sería valioso que consideraran lo que pasa en terreno" (Entrevistado 5)

\section{DISCUSIÓN}

La implementación de una política pública implica poner en marcha múltiples acciones políticas y administrativas que se soporten mutuamente (15). Es importante que los actores involucrados en su desarrollo no solo sean representantes gubernamenta- les, sino que también, se considere la participación de otros componentes involucrados de la Sociedad Civil (16). En el caso de la UCF, destaca la fuerte participación de un actor particular, representado en la fundadora de la unidad, quien es considerada por los participantes como una pieza clave en el éxito de la implementación, sumado al fuerte compromiso social y motivación del resto del equipo.

El liderazgo es uno de los factores más determinantes e influyentes en la percepción que tienen los trabajadores del clima organizacional (17), coincidiendo con lo observado en la Unidad Clínico Forense, como un equipo funcional y bien cohesionado. Asimismo, en todo proceso de cambio los líderes se transforman en una pieza clave para participar y potenciar el aprendizaje continuo, tanto el propio como el de sus colaboradores (18). En la UCF esto se ve reflejado en la participación de sus integrantes en capacitaciones asociadas al quehacer de la unidad, viniendo a sumarse esto último a los potenciadores del éxito de su implementación. Con respecto al ámbito psicológico del clima organizacional, se debe tener en cuenta que los prestatarios que brindan atención a víctimas/sobrevivientes de violencia sexual se encuentran expuestos a sufrir consecuencias como el síndrome de burnout, la traumatización vicaria y/o la movilización de sus propias experiencias de violencia $(19,20)$. Los resultados evidencian que dentro de la UCF utilizan distintos métodos de autocuidado que provienen desde la autogestión, como las jornadas de autocuidado y defusing, siendo este último una técnica de desactivación inmediata post emergencia que consta de una reunión informal donde participan todos aquellos implicados en la emergencia (21).

Sobre los elementos dificultantes, destacaba la percepción de actitudes hostiles desde otras unidades del hospital, asociadas la subestimación de la importancia de tener un equipo exclusivo abocado a la atención de víctimas de violencia sexual, lo cual pese a ser un modelo único de UCF en Chile, en este caso asegura el cumplimiento de los objetivos de la norma. Este hallazgo es coincidente a lo que se describe ante la existencia de varios agentes involucrados en la implementación de una política pública; donde existe mayor dificultad para lograr el consenso en los objetivos y metas, dificultando el proceso y resultados (15). 
Para asegurar que una política se cumpla exitosamente es necesario que la directriz sea conocida, aceptada y adoptada por los actores sociales (22). La falta de difusión hacia el público fue considerado por los entrevistados como problemática al limitar la asistencia de usuarios por desconocimiento. En el caso de las políticas públicas, la propaganda involucra acciones realizadas por actores políticos con el fin de impulsar la adhesión a un sistema de normas y asegurar su aprobación y cumplimiento por la población (22).

\section{CONCLUSIONES}

Los miembros de la UCF experimentaron un complejo proceso de implementación debido a diversos factores dificultantes, asociados principalmente a una respuesta insuficiente de los agentes de gestión. Sin embargo, es percibido como un proceso exitoso, destacando la autogestión, el liderazgo y la motivación personal como factores determinantes para su adecuada instauración. Por otro lado, consideramos importante cuestionar e investigar en profundidad cómo estos factores dificultantes ponen en riesgo el éxito de una norma gubernamental fundamental para dar respuesta al fenómeno de la violencia sexual y además, a la salud emocional de sus funcionarios.

\section{REFERENCIAS}

1. García-Moreno C., Guedes A. Comprender y abordar la violencia contra las mujeres. Violencia sexual [Internet]. Washington, DC; 2013 [Consultado el 7 de agosto de 2018].

2. Janos E, Espinosa A. Representaciones sociales sobre roles de género y su relación con la aceptación de mitos y creencias sobre la violencia sexual. Revista Límite [Internet]. 2015 [Consultado el 24 noviembre de 2019]; 10(33).

3. Mahalik J, Locke B, Ludlow L, Diemer M, Scott $\mathrm{R}$, Gottfried M et al. Development of the Conformity to Masculine Norms Inventory. Psychology of Men \& Masculinity [Internet]. 2003 [Consultado el 25 de agosto de 2020];4(1):3-25. Disponible en: https://doi. org/10.1037/1524-9220.4.1.3

4. MINSAL. Guía Clínica: Atención de Niños, Niñas y Adolescentes Menores de 15 años, Víctimas de Abuso Sexual [Internet]. Santiago; 2011 [Consultado el 7 de agosto de 2018]. Disponible en: $\quad$ http://www.minsal.cl/portal/url/item/aaa27720f363a745e04001011e011120.pdf
5. GfK Adimark. "Encuesta Nacional de Victimización por Violencia Intrafamiliar y Delitos Sexuales" [Internet]. 2013 [Consultado el 7 de agosto de 2018]. Disponible en: http://www.seguridadpublica.gov.cl/ filesapp/Presentacion\%20VIF_adimark_final.pdf

6. Alcázar M., Duque C., López J., Muñoz P., Orellana C., Pesse F., et al. Norma general técnica para la atención de víctimas de violencia sexual [Internet]. Santiago: SML; 2016 [Consultado el 7 de agosto de 2018]. Disponible en: http://icmer.org/adjuntos/ NT_ATENCION_VICTIMAS-SML.pdf

7. Montoya S David, Díaz S Ricardo, Reyes O Fernando, Abusleme A Carlos, Garrido C Jaime. Peritaje médico legal en delitos sexuales: una pauta práctica para su correcta realización. Rev. chil. obstet. ginecol. [Internet]. 2004 [Consultado el 05 de enero de 2019]; 69(1): 55-59. Disponible en: https://scielo. conicyt.cl/scielo.php?script=sci_arttextEpid=S071775262004000100012\&lng=es. http://dx.doi. org/10.4067/S0717-75262004000100012.

8. Alarcón C. Implementación territorial de las salas de acogida para la atención a víctimas de violencia sexual: ¿Es posible que las instituciones médicas reduzcan la victimización secundaria?. CMS [Internet]. 2019 [Consultado el 23 noviembre de 2019 ;58(2):57-62. Disponible en: http://cms.colegiomedico.cl/wp-content/uploads/2018/08/12.-CMS-VOL58-N\%C3\%82\%C2\%B02.-Alarc\%C3\%83\%C2\%B3nC..pdf

9. Tapia M, Campillo B, Cruickshank S, Morales G. Manual de Incidencia en políticas públicas. 1ra ed. Alternativas y Capacidades Ciudad de México; 2010.

10. Neira, R. Evaluación del Bienestar materno de mujeres en situación de parto de dos Hospitales del SNSS, Región Metropolitana, Chile, 2010. [Documento PDF].

11. Consejo Nacional de la Infancia. Unidades Clínico Forense [Folleto]. Santiago, Chile 2014 [Consultado el 5 de enero de 2019]. Disponible en: $h t t p: / / w w w$. hospitalfricke.cl/wp- content/uploads/2017/12/FOLLETO_FORENSES_FINAL01.pdf

12. Hospital Dr. Gustavo Fricke. Quiénes somos [Internet]. 2014 [Consultado el 5 de enero de 2019]. Disponible en: http://www.hospitalfricke.cl/?page_ $i d=83$

13. Hospital Dr. Gustavo Fricke. Unidad Clínico Forense [Internet]. 2017 [Consultado el 5 de enero de 2019]. Disponible en: $h t t p: / / w w w . h o s p i-$ talfricke.cl/?page_id $=8547$ 
14. Hernandez R, Fernández C, Baptista M. Metodología de la investigación. 6th ed. Caracas:

McGraw Hill; 2013.

15. Molina G, Cabrera G. Políticas públicas en salud: aproximación a un análisis. Fleisacher E, editor. Colombia: Facultad Nacional de Salud Pública "Héctor Abad Gómez", Universidad de Antioquia; 2008. p. 5-12.

16. Rodríguez JM. Políticas públicas. Rev Venez Enfermería [Internet]. 2017, [Consultado el 07 de junio de 2019]; 4(2):17-26. Disponible en: http://190.169.30.98/ojs/index.php/rev_venf/article/view/15917

17. Ponce, P., Pérez, S., Cartujano, S., López, R., Álvarez, C., \& Real, B.. Liderazgo femenino y clima organizacional, en un instituto universitario. In Global Conference on Business and Finance Proceedings. 2014 [Consultado el 5 de julio de 2019]; 9(1):1031-1036.

18. Palomo M. Liderazgo y motivación de equipos de trabajo. 8th ed. Madrid: ESIC; 2019.

19. Ojeda T. El autocuidado de los profesionales de la salud que atienden a víctimas de violencia sexual. Revista Peruana de Ginecología y Obstetricia. 2006 [Consultado el 5 de julio de 2019];52(1):21-27. Disponible en: http://spog.org.pe/web/revista/index.php/RPGO/article/view/347/319

20. Puhl, S, Izcurdia, M., Oteyza, G, Escayol, M. Síndrome de Burnout en profesionales que asisten a víctimas de violencia familiar. Anuario de Investigaciones [Internet]. 2016, [Consultado el 5 de julio de 2019]; 23:229-236. Disponible en: http://www.redalyc.org/articulo.oa?id=369152696023

21. Universitat Autònoma de Barcelona. Primeros Auxilios Psicológicos (PAP) [Internet]. 2015 [Consultado el 25 noviembre de 2019]. Disponible en: https:// es.coursera.org/lecture/pap/v5-4-la-tecnica-deldefusing-explicacion-BBc9c

22. Gómez R. Gestión de políticas públicas: aspectos operativos. Rev Fac. Nac. Salud Pública [Internet]. 2012 [Consultado el 07 de junio de 2019]; 30(1): 223-36. Disponible en: http://www.scielo.org. co/pdf/rfnsp/v30n2/v30n2a11.pdf 